Www.jmscr.igmpublication.org

Index Copernicus Value: 79.54

ISSN (e)-2347-176x ISSN (p) 2455-0450

crossref DOI: https://dx.doi.org/10.18535/jmscr/v7i5.126

Journal Of Medical Science And Clinical Research

IGM Publication

An official Publication of IGM Publication

\title{
A Descriptive Analysis of Prescribing Patterns of Drugs in Chronic Kidney Disease Patients on Maintenance Hemodialysis
}

Authors

Chandel Ritesh Kumar', Bhargava Jyotsna ${ }^{2 *}$, Dadheech Jaya ${ }^{3}$

${ }^{1,3}$ Postgraduate Student, ${ }^{2} \mathrm{Sr}$. Professor

Department of Pharmacology, SMS Medical College, Jaipur, Rajasthan, India

Corresponding Author

Bhargava Jyotsna

Sr. Professor, Department of Pharmacology, SMS Medical College, Jaipur, Rajasthan, India

\begin{abstract}
Introduction: Chronic kidney disease is a global threat to health particularly for developing countries because of its increasing incidence, poor outcome and high cost of treatment. The treatment includes dialysis and renal transplantation. Appropriate drug selection for patients with chronic kidney disease $(C K D)$ is important in order to avoid unwanted drug effects and to ensure optimal patient outcomes. Rational drug prescription is a difficult task in CKD patients. So, present study was planned to know the prescribing pattern of drugs in chronic kidney disease on maintenance hemodialysis at Jaipur, Rajasthan.

Materials and Methods: The study was carried out in Department of Pharmacology in association with Department of Nephrology, S.M.S. Medical College \& Hospital, Jaipur (Rajasthan) from April 2017 to March 2018. Socio-demographic profile and details of drugs administered were recorded in a predesigned and pretested proforma. Results were expressed as percentages and proportions. Pearson's correlation coefficient ( $r$ ) was calculated to show correlation between drug-drug interaction and drug per prescription.

Results: In the present study, out of160 chronic kidney patients majority were males $(75.63 \%)$ with mean age of $39.5 \pm 13.29$ years and $24.37 \%$ were females with mean age of $40.41 \pm 13.53$ years. Co-morbidities were anemia (98.13\%), hypertension (95.65\%), infections (22.5\%) and diabetes mellitus (6.85\%). Mean number of drugs per prescription were $7.84 \pm 1.40$. Majority of prescriptions $(86.87 \%)$ contained 6 to 9 drugs. Most common drugs prescribed were vitamins and minerals (32\%) followed by cardiovascular drugs (21.03\%) and hematopoietic drugs (14.58\%). Majority of prescription (75.62\%) contained 3-5 drug-drug interactions (DDIs) and the number of DDIs increased with increasing number of drugs per prescription $(r$ $=0.2606, p$ value $<0.001$ )
\end{abstract}

Conclusion: Certain areas of concern such as polypharmacy, underuse of erythropoietin stimulating agents and calcium free phosphate binders and high incidence of potential DDIs were addressed in the present study. There is a need for exploring these issues in detail through future drug use studies.

Keywords: Chronic Kidney Disease, Maintenance Hemodialysis, Prescribing Patterns.

\section{Introduction}

Chronic kidney disease (CKD) is a general term for heterogeneous disorders affecting kidney structure and its function. It is defined as either kidney damage or a decreased glomerular filtration rate of less than $60 \mathrm{~mL} / \mathrm{min} / 1.73 \mathrm{~m}^{2}$ for 3 
or more months. ${ }^{1}$ CKD is a key determinant of poor health outcomes for major noncommunicable diseases, and it is a worldwide threat to public health. Estimates of the global burden of the diseases reported that kidney and urinary tract diseases contribute $8,30,000$ deaths annually making them the $12^{\text {th }}$ highest cause of death (1.4\% of all deaths) and disability-adjusted life years (DALY) in $1,88,67,000$, i.e. $17^{\text {th }}$ cause of disability ( $1 \%$ of all DALY). ${ }^{2}$

In India, the prevalence of CKD was observed to be $17.2 \%$ and about $6 \%$ had stage 3 or above among them. It is more common among males compared to females due to stress, alcoholism, hypertension, diabetes mellitus, smoking and cumulative risk factors of chronic vascular disease (CVD). ${ }^{3}$

Appropriate drug selection for patients with chronic kidney disease (CKD) is important in order to avoid unwanted drug effects and to ensure optimal patient outcomes. Rational drug prescription is a difficult task in CKD patients. These patients are at higher risk of drug related problems since they need complex therapeutic regimens that require frequent monitoring and dosage adjustments. In addition, they usually have other co-morbidities. ${ }^{4}$ Inappropriate use of medications can increase adverse drug effects, which can be reflected by excessive length of hospital stays, excessive health care utilization and costs. ${ }^{5}$ As CKD progresses, the drugs prescribed to these patients also increases and the prevalence of drug related problems also increases. ${ }^{6}$ Dialysis patients require special consideration regarding prescription of drugs because of their altered pharmacokinetic and pharmacodynamics profiles.

Drug utilization studies were conducted frequently all over the world; however, in India, there is no clear picture of overall medication profile in CKD patients specially in Rajasthan. The present study was conducted to find out the prescribing pattern of drugs in chronic kidney disease patients on maintenance hemodialysis which will be helpful in generating data to evaluate, monitor and if possible, suggest modifications in prescribing practices to make medical care rational in hemodialysis patients.

\section{Materials and Methods}

A cross sectional observational study wascarried out in Department of Pharmacology and Department of Nephrology, SMS Medical College, Jaipur (Rajasthan) from April 2017 to March 2018. Prior approval from institutional ethics committee was taken. After taking informed consent eligible patients undergoing hemodialysis in Nephrology Department of the institute were recruited for the study. Total 160 CKD patients were interviewed. Sociodemographic data like age, sex, residence, socioeconomic status, educational and occupational status were noted in a pre-designed and pre-tested performa. Details about final diagnosis, associated co-morbidities, drugs prescribed were recorded.

The data collected were entered in Microsoft Excel 2010 Spreadsheets and analyzed using MedCalc Statistical Software version 17.2. Results were expressed as percentages and proportions. Pearson's correlation coefficient (r) was calculated to show correlation between drugdrug interaction and drug per prescription.

\section{Results}

Out of 160 study participants, $121(75.63 \%)$ were males and 39 (24.37\%) were females. Majority of patients were in age group of 21-30 years (27.5\%). Only $5 \%$ patients were in age group of 20 years and below. $21.25 \%$ of patients were in age group of $31-40$ years, $24.4 \%$ of patients were in age group of $41-50$ years, $14.4 \%$ were in age group of 51-60 years and $7.5 \%$ patients were in age group more than 60 years. Mean age of males was $39.5 \pm$ 13.29 years while mean age of females was 40.41 \pm 13.53 years.

53 patients $(33.13 \%)$ belonged to middle socioeconomic class while $107(66.88 \%)$ belonged to lower socio-economic class.25 patients (15.63\%) were illiterate, $59(36.9 \%)$ had primary level education, 34 (21.25\%) had secondary level 
education, 21 (13.13\%) had senior secondary level education and $21(13.13 \%)$ had graduation \& above level education.

The employed status of study participants was observed and it was found that $127(79.37 \%)$ were employed, while 33 (20.63\%) were unemployed. Among the employed, 45 (28.13\%) were semiprofessionals, 35 (21.87\%) were homemakers, 30 $(18.75 \%)$ were semi-skilled workers, $9(5.63 \%)$ were unskilled workers and $8(5 \%)$ were skilled workers.

Most common associated comorbidity was anemia $(98.13 \%)$ followed by hypertension (95.63), infections (22.5\%), diabetes mellitus (6.85\%), hypothyroidism $(2.5 \%)$ and others $(3.75 \%)$ (Table-1). It was observed in present study that about $6(3.76 \%)$ prescriptions contained up to 5 drugs, 139 (86.87\%) prescription contained 6-9 drugs and $15(9.37 \%)$ prescriptions contained 1013 drugs. Total 1255 drugs were prescribed for 160 patents (Table-2).

31 (19.38\%) prescriptions contained 0-2 drugdrug interactions (DDIs), 121(75.62\%) prescriptions contained 3-5 DDIs and only 1 $(0.63 \%)$ prescription contained > 5 DDIs (Figure1).Most common drugs prescribed were vitamins and minerals $402(32.03 \%)$ followed by cardiovascular (CVS) drugs264 (21.03\%), hematopoetic drugs 183 (14.58\%), gastrointestinal (GI) drugs 178 (14.18) and blood alkalizers 115 (9.16\%) (Table-3).

The relation between drug-drug interaction and drug per prescription is expressed in the following regression equation:

DDI $=0.2147 \mathrm{x}+1.4848$

There was a strong correlation between drug-drug interaction and drug per prescription $(\mathrm{p}<0.001$, r:0.2606) (Table-4 \&Figure-2).

Majority of patients were highly adherent to therapy $130(81.25 \%)$ while $30(18.75 \%)$ of patients showed medium adherence to therapy (Figure-3).
Table-1: Distribution of study subjects according to associated comorbidities

\begin{tabular}{|l|c|c|}
\hline Associated Comorbidities & $\begin{array}{c}\text { Number } \\
\text { (n) }\end{array}$ & $\begin{array}{c}\text { Percentage } \\
(\mathbf{\%})\end{array}$ \\
\hline Anemia & 157 & 98.13 \\
\hline Hypertension & 153 & 95.63 \\
\hline Infections & 36 & 22.50 \\
\hline Diabetes Mellitus & 11 & 6.88 \\
\hline Hypothyroidism & 4 & 2.50 \\
\hline Other & 6 & 3.75 \\
\hline
\end{tabular}

Multiple responses

Table-2: Distribution of study subjects according to drugs per prescription

\begin{tabular}{|l|c|c|c|c|}
\hline \multirow{2}{*}{$\begin{array}{l}\text { Drugs Per } \\
\text { Prescription }\end{array}$} & \multicolumn{2}{|c|}{ CKD Patients } & \multicolumn{2}{c|}{$\begin{array}{c}\text { Drugs } \\
\text { Prescribed }\end{array}$} \\
\cline { 2 - 5 } & $\mathbf{( n )}$ & $\mathbf{( \% )}$ & $\mathbf{( n )}$ & $\mathbf{( \% )}$ \\
\hline 4 & 1 & 0.63 & 4 & 0.32 \\
\hline 5 & 5 & 3.13 & 25 & 1.99 \\
\hline 6 & 16 & 10 & 96 & 7.65 \\
\hline 7 & 43 & 26.87 & 301 & 23.98 \\
\hline 8 & 50 & 31.25 & 400 & 31.87 \\
\hline 9 & 30 & 18.75 & 270 & 21.51 \\
\hline 10 & 11 & 6.87 & 110 & 8.76 \\
\hline 11 & 1 & 0.63 & 11 & 0.88 \\
\hline 12 & 1 & 0.63 & 12 & 0.96 \\
\hline 13 & 2 & 1.25 & 26 & 2.07 \\
\hline Total & $\mathbf{1 6 0}$ & $\mathbf{1 0 0 . 0 0}$ & $\mathbf{1 2 5 5}$ & $\mathbf{1 0 0 . 0 0}$ \\
\hline
\end{tabular}

Table-3: Distribution of Various Group of Drugs Prescribed

\begin{tabular}{|l|c|c|}
\hline Drugs & Number & Percentage \\
\hline Cardiovascular Drugs & 264 & 21.03 \\
\hline Gastrointestinal Drugs & 178 & 14.18 \\
\hline Anti Diabetic Drugs & 7 & 0.56 \\
\hline Hematopoietic Drugs & 183 & 14.58 \\
\hline Phosphate Binders & 22 & 1.75 \\
\hline Vitamin and Minerals & 402 & 32.03 \\
\hline Antibiotics & 59 & 4.70 \\
\hline Blood Alkalizer & 115 & 9.16 \\
\hline Other & 25 & 1.99 \\
\hline Total & $\mathbf{1 2 5 5}$ & $\mathbf{1 0 0 . 0 0}$ \\
\hline
\end{tabular}


Table 4: Correlation of DDIs with Drugs per Prescription

\begin{tabular}{|c|c|c|c|c|c|c|c|c|c|c|c|c|c|c|c|c|}
\hline \multirow{3}{*}{$\begin{array}{l}\text { Drugs/ } \\
\text { Prescription }\end{array}$} & \multicolumn{16}{|c|}{ Drug-Drug Interaction } \\
\hline & \multicolumn{2}{|c|}{$\mathbf{0}$} & \multicolumn{2}{|r|}{$\mathbf{1}$} & \multicolumn{2}{|c|}{2} & \multicolumn{2}{|c|}{3} & \multicolumn{2}{|c|}{4} & \multicolumn{2}{|c|}{5} & \multicolumn{2}{|c|}{6} & \multicolumn{2}{|c|}{ Total } \\
\hline & $\mathbf{n}$ & $\%$ & $\mathbf{n}$ & $\%$ & $\mathbf{n}$ & $\%$ & $\mathbf{n}$ & $\%$ & $\mathbf{n}$ & $\%$ & $\mathrm{n}$ & $\%$ & $\mathbf{n}$ & $\%$ & $\mathrm{n}$ & $\%$ \\
\hline 4 & 0 & 0.00 & 1 & 0.63 & 0 & 0.00 & 0 & 0.00 & 0 & 0.00 & 0 & 0.00 & 0 & 0.00 & $\mathbf{1}$ & 0.63 \\
\hline 5 & 1 & 0.63 & 2 & 1.25 & 1 & 0.63 & 1 & 0.63 & 0 & 0.00 & 0 & 0.00 & 0 & 0.00 & 5 & 2.50 \\
\hline 6 & 2 & 1.25 & 1 & 0.63 & 2 & 1.25 & 7 & 4.38 & 4 & 2.50 & 0 & 0.00 & 0 & 0.00 & 16 & 10.00 \\
\hline 7 & 0 & 0.00 & 4 & 2.50 & 1 & 0.63 & 21 & 13.13 & 17 & 10.63 & 0 & 0.00 & 0 & 0.00 & 43 & 26.88 \\
\hline 8 & 0 & 0.00 & 5 & 2.50 & 3 & 1.88 & 12 & 7.50 & 28 & 17.50 & 2 & 1.25 & 0 & 0.00 & 50 & 31.25 \\
\hline 9 & 0 & 0.00 & 3 & 1.88 & 2 & 1.25 & 11 & 6.88 & 13 & 8.13 & 1 & 0.63 & 0 & 0.00 & 30 & 18.75 \\
\hline 10 & 0 & 0.00 & 1 & 0.63 & 1 & 0.63 & 2 & 1.25 & 4 & 2.50 & 2 & 1.25 & 1 & 0.63 & 11 & 6.88 \\
\hline 11 & 0 & 0.00 & 0 & 0.00 & 0 & 0.00 & 0 & 0.00 & 0 & 0.00 & 1 & 0.63 & 0 & 0.00 & 1 & 0.63 \\
\hline 12 & 1 & 0.63 & 0 & 0.00 & 0 & 0.00 & 0 & 0.00 & 0 & 0.00 & 0 & 0.00 & 0 & 0.00 & $\mathbf{0}$ & 0.00 \\
\hline 13 & 0 & 0.00 & 0 & 0.00 & 0 & 0.00 & 1 & 0.63 & 0 & 0.00 & 1 & 0.63 & 0 & 0.00 & 2 & 1.25 \\
\hline Total & 4 & 2.50 & 17 & 10.63 & 10 & 6.25 & 55 & 34.38 & 66 & 41.25 & 7 & 4.38 & 1 & 0.63 & 160 & 100 \\
\hline
\end{tabular}

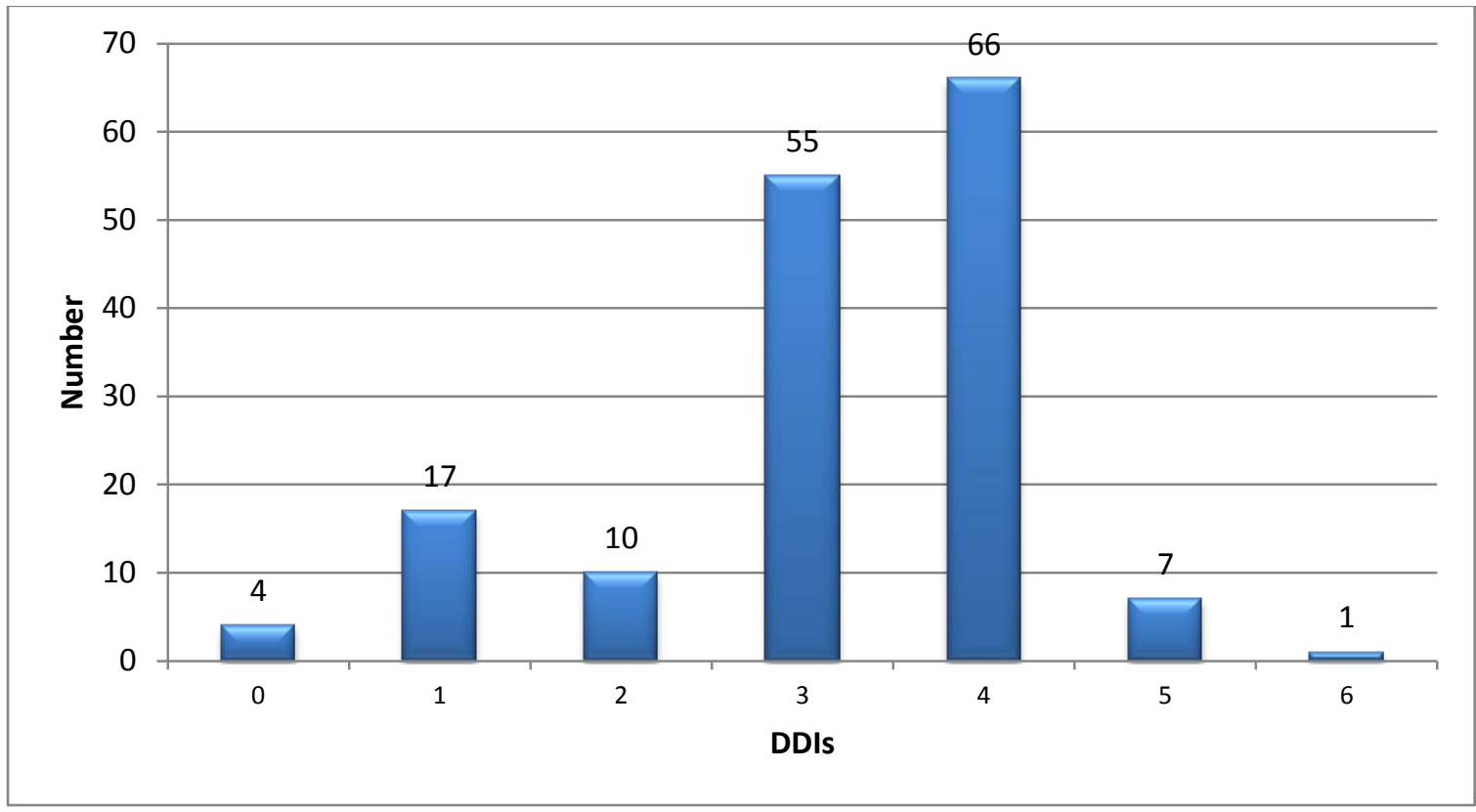

Figure-1: Distribution of study subjects according to drug-drug interactions

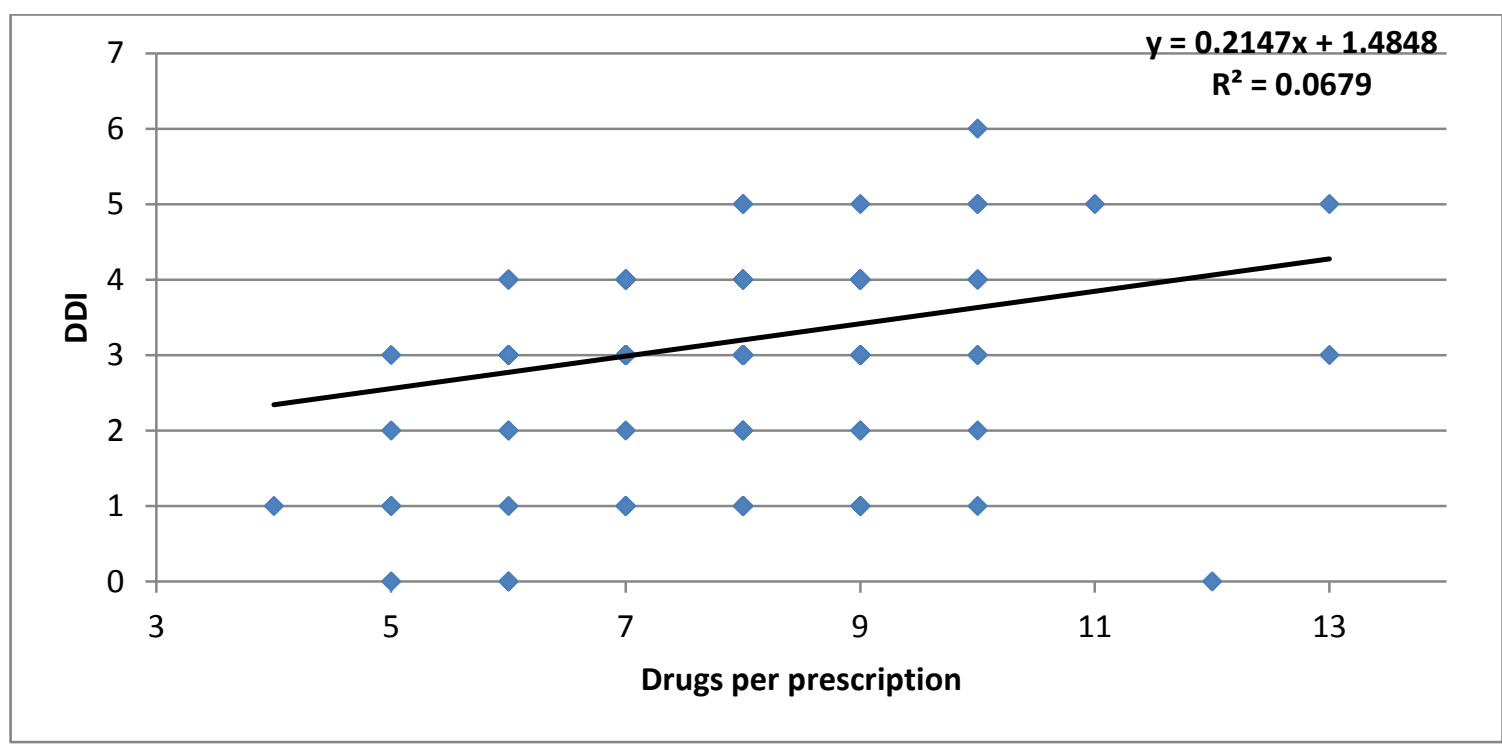

Figure-2: Correlation of DDIs with Drugs per Prescription 


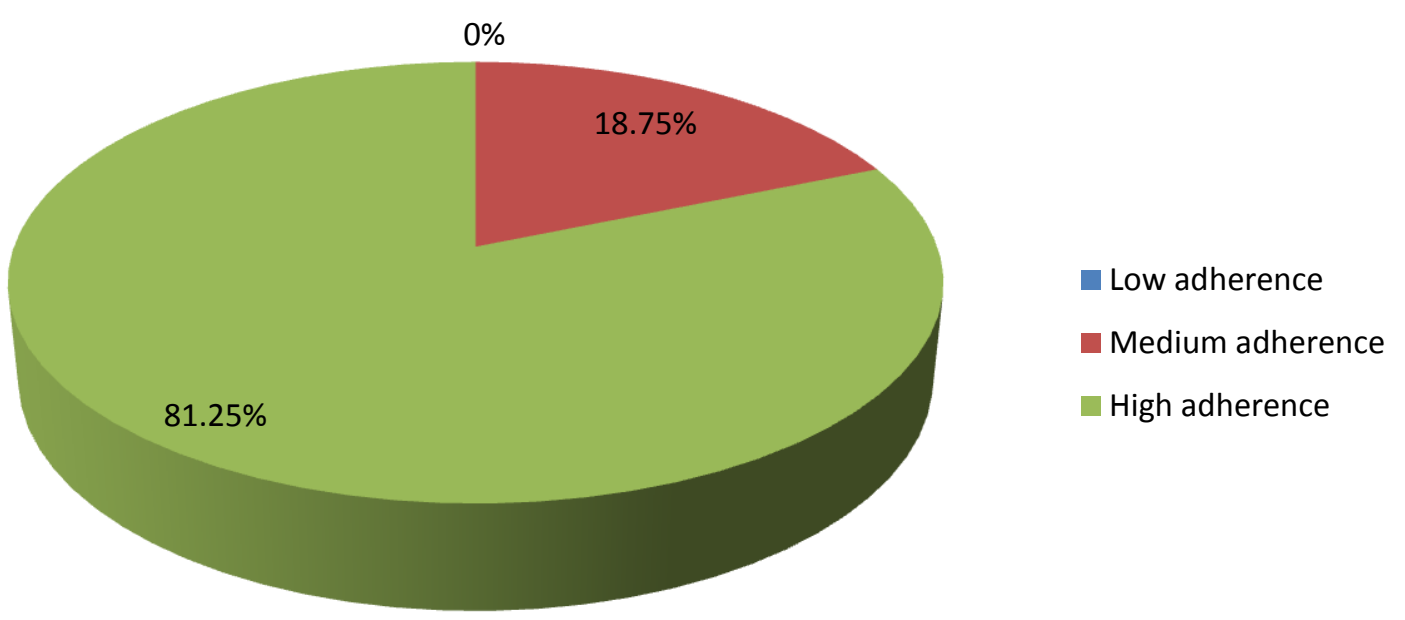

Figure-3: Distribution of patient's adherence to therapy according to Morisky Medication Adherence Scale

\section{Discussion}

Chronic kidney disease (CKD) has numerous causes. The most common causes of CKD are diabetes mellitus and long-term, uncontrolled hypertension. ${ }^{7} \quad$ Polycystic kidney disease is another well-known cause of CKD. The majority of people afflicted with polycystic kidney disease have a family history of the disease. Other genetic illnesses affect kidney function, as well. Overuse of common drugs such as ibuprofen, and acetaminophen (paracetamol) can also cause chronic kidney disease. ${ }^{8}$ Some infectious disease agents, such as hantavirus, can attack the kidneys, causing kidney failure. ${ }^{9}$

The present study showed male predominance $75.3 \%$, and mean age to be 40 years which was similar to previous studies. ${ }^{10-11}$ This highlighted that males were more prone to develop CKD than females because of formers association with risk factors like smoking, alcohol, hypertension (HTN) and hyperlipidemia. It was highlighted by present study that CKD was common in middle and low socio-economic classes and majority of sufferers were illiterates or primary educated, although most of them were employed. No comparable data were found in previous studies.

The mean number of drugs per prescription was $7.84 \pm 1.4, \quad$ suggestive of polypharmacy.
Polypharmacy is defined as use of 5 or more drugs to a patient at a time. However, in view of complex nature of the disease and coexisting morbidities of CKD, few researchers have suggested the use of more than 9 drugs at a time to be considered as polypharmacy. ${ }^{12}$ The result of present study was similar to previous studies that found the mean number to be 7.2, 7.49 and 7.87 respectively. ${ }^{13-15}$

Most common drugs prescribed in this study were vitamins and minerals (32.03\%) followed by cardiovascular drugs (21.03\%), hematopoetic drugs (14.58\%), gastrointestinal drugs (14.18\%) and blood alkalizers $(9.16 \%)$. These finding were similar to another study from India where most common drugs prescribed were vitamin and minerals $(24.71 \%)$ followed by cardiovascular drugs (22.14\%) and cematopoetic drugs $(2.15 \%) .{ }^{16}$ However results were differed from other studies where most common prescribed drugs were CVS drugs $(42 \%)^{10}$, calcium and phosphate metabolism $(88 \%)^{13}$ and Calcium carbonate $(77.1 \%)^{14}$.

In spite of high prevalence of anemia (98.13\%), erythropoietin was under prescribed. In the present study erythropoietin stimulating agent (ESA) was used along with oral iron folic acid (IFA) in $34(21.65 \%)$ of anemic patients. In rest of 
anemic patients only oral IFA was used. This was lower than other studies $(60 \%)^{13}$ and higher than other studies where ESAs were not used and anemic patients were treated only with oral iron preparations. ${ }^{10,11}$

Among 11 diabetes mellitus patients only 7 (63\%) received antidiabetic medications. Insulin was used in $4(57 \%)$ and oral Teneligliptin was used in $3(43 \%)$ diabetic patients. These findings strongly support hypothesis that requirement of antidiabetic medication falls in CKD patients. The results were similar to previous studies.$^{11,14}$

Hypertension was the second most common comorbidity in the study $(95.63 \%)$. Among the drugs to treat hypertension most commonly used were calcium channel blockers (11.55\%), followed by alfa-2 agonists (4.71\%), diuretics (3.35\%), beta-blockers $(0.96 \%)$ and alfa-blockers $(0.49 \%)$. The result was contradictory to other studies where among cardiovascular drugs, diuretics $^{10,16}$ and Beta-blockers ${ }^{15}$ were the most commonly prescribed anti hypertensives.

Hyperphosphatemia is among the most common metabolic complications of end stage renal disease. Large observational studies had identified hyperphosphatemia as an independent risk factor for cardiovascular disease and mortality on dialysis. Administration of dietary phosphorus binders to block intestinal phosphorus absorption is the cornerstone of therapy for hyperphosphatemia. ${ }^{17}$ Of all prescribed drugs $1.99 \%$ were phosphate binders. Sevelemar (1.19\%) was most frequently prescribed phosphate binder followed by calcium acetate $(0.48 \%)$ and lanthanum carbonate $(0.08 \%)$. Results of this study differs from other studies ${ }^{13,14}$ where calcium carbonate was the most commonly prescribed phosphate binder. The reason behind under use of Sevelemar and other calcium free phosphate binders in present study and in previous studies was their higher cost.

Antibiotics are required in hemodialysis patients for reasons such as infection arising from vascular access, urinary or respiratory tract infections and sepsis. Total antibiotics prescribed in present study were $5.33 \%$ which were used in patients having different kind of infective conditions $(22.5 \%)$. These antibiotics were used only when clear indication of infection was present, showing judicious use of antibiotics by the treating physicians. Most commonly used group of antibiotics was fluoroquinolones (1.82\%), followed by cephalosporins (1.28\%). Antibiotics must be used when there is presence of infection or prophylactically to prevent the infections. One of the previous studies ${ }^{18}$ showed irrational use of antibiotics as (82\%) of patients were prescribed antibiotics with no clear indication for prescribing them.

The number of prescriptions with potential drugdrug interactions (DDIs) in this study was high $(97.5 \%)$. This was higher than other studies $(89.1 \%)^{14},(76.09 \%)^{15},(95.9 \%)^{16},(82.1 \%)^{18}$. The number DDIs increased with increase in number of drugs per prescription. The mean number of DDIs per prescription in our study were $3.17 \pm$ 1.16. This was lower than previous study $15.69 \pm$ $11^{16}$ and higher than other studies $(2.7)^{15},(2)^{18}$. Some of the potentially interacting combinations noted in our study were, Iron folic acid + Calcium, Iron folic acid + Proton pump inhibitors, Sodium bicarbonate + Iron folic acid, Nifedipine + Calcium.

Adherence is a major concern in the therapy of CKD. World Health Organization estimates that only half of people with chronic diseases take their medications consistently as prescribed. Nonadherence to medication can be harmful as well as expensive for patients. In the present study adherence level was high in $130(81.25 \%)$ patients and medium in $30(18.75 \%)$ patients. Number of patients with high adherence in this study was lower than previous study $(93.6 \%)^{19}$, and higher than previous studies $(7.3 \%)^{20},(28.09 \%)^{21}$, $(66.2 \%)^{22}$ while number of patients with medium adherence in present study was lower than previous studies $(55.3 \%)^{20},(40.45 \%)^{21}$.Patients with low adherence were not recorded in the study, whereas in previous studies low adherence was found to be higher $(6.4 \%)^{19},(37.3 \%)^{20}$ 
and $(33.8 \%)^{22}$. The probable reason for high adherence to therapy in our study was positive physician-patients relationship, and availability of required medicines for free from the hospital.

\section{Conclusion}

The present study is limited by its sample size, unicentric nature and hospital-based evaluation. However, it had generated a profile of drug use in Indian CKD patients on hemodialysis that can serve as the basis for future comparisons. The study may also help to identify educational and quality improvement opportunities to prevent medication-related problems in this population. It had identified certain areas of concern such as polypharmacy, underuse of ESAs and calcium free phosphate binders, high incidence of potential DDIs and under evaluation and treatment of mental health conditions in hemodialysis patients.

\section{References}

1. Levey AS, Coresh J. Chronic kidney disease. Lancet. 2012;379:165-80.

2. Couser WG, Remuzzi G, Mendis S, Tonelli M. The contribution of chronic kidney disease to the global burden of major noncommunicable diseases. Kidney Int. 2011;80:1258-70.

3. Rani NV, Thomas R, Rohini E, Soundararajan P, Kannan G, Thennarasu P. A study on drug related problems in chronic kidney disease patients of a tertiary care teaching hospital in South India. World J Pharmaceu Res. 2014;3:1403-17.

4. RFunfstuck, Ott U, Naber KG. The interaction of urinary tract infection and renal insufficiency. International Journal of Antimicrobial Agents. 2006;72-77.

5. Bonk ME, Krown H, Matuszewski K, Oinonen M. Potentially inappropriate medications in hospitalized senior patients. Am J Health Syst Pharm. 2006;63:1161-5.

6. Veerappan I, Abraham G. Chronic kidney disease: current status, challenges and management in India. Available from http://www.apiindia.org/

medicine_update_2013/chap 130.

7. Kes P, Basic-Jukic N, Ljutic D, BrunettaGavranic B. The role of arterial hypertension in developement of chronic renal failure. Acta Med Croatica 2011;65 (Suppl 3):78-84.

8. Perneger TV, Whelton PK, Klag MJ. Risk of Kidney Failure Associated with the Use of Acetaminophen, Aspirin, and Nonsteroidal Antiinflammatory Drugs. N Engl J Med. Dec 1994;331(25):1675-79.

9. Appel, Gerald B; Mustonen, Jukka. Renal involvement with hantavirus infection (hemorrhagic fever with renal syndrome). UpToDate. 2012; Retrieved 1 January 2013.

10. Narayana Murthy BV, Satyanarayana V. Prescribing pattern of drugs in chronic kidney disease patients on hemodialysis at a tertiary care hospital. IJBCP. 2017;6(4);928-32.

11. Tamiselvan T, Veerapandiyan AK, Kathik N. Study on drug utilization pattern of chronic renal failure patients in a tertiary care hospital. International Journal of Pharmacy and Pharmaceutical Sciences. ISSN- 0975-1491.

12. Bushardt RL, Massey EB, Simpson TW, Ariail JC, Simpson KN. Polypharmacy: Misleading, but manageable. Clin Interv Aging. 2008;3:383- 9.

13. Tozawa $\mathrm{M}$, Iseki $\mathrm{K}$, Iseki $\mathrm{C}$, Oshiro $\mathrm{S}$, Higashiuesato $\mathrm{Y}$, Yamazato $\mathrm{M}$, et al. Analysis of drug prescription in chronic haemodialysis patients. Nephrol Dial Transplant. 2002;17:1819- 24.

14. Al-Ramahi R, Raddad AR, Rashed AO, Bsharat A, Abu-Ghazaleh D, Yasin E, Shehab O. Evaluation of potential drugdrug interactions among Palestinian hemodialysis patients. BMC Nephrology. BMC series - open, inclusive and trusted 201617:96. 
15. Rama M, Viswanathan G, Acharya LD, Attur RP, Reddy PN, Raghavan SV. Assessment of Drug-Drug Interactions among Renal Failure Patients of Nephrology Ward in a South Indian Tertiary Care Hospital Indian J Pharm Sci. 2012 Jan-Feb; 74(1): 63-68.

16. Fasipe OJ, Akhideno PE, Nwaiwu O, Adelosoye AA. Assessment of prescribed medications and pattern of distribution for potential drug-drug interactions among chronic kidney disease patients attending the Nephrology Clinic of Lagos University Teaching Hospital in Sub-Saharan West Africa. October 2017; 9:125-132.

17. Isakova T. Phosphorus Binders and Survival on Hemodialysis. J Am Soc Nephrol. 2009 Feb; 20(2):388-396.

18. Chakraborty S, Ghosh S, Banerjea A, De RR, Hazra A, Mandal SK. Prescribing patterns of medicines in chronic kidney disease patients on maintenance hemodialysishttp://www.ijponline.com,October 26, 2016.

19. Silvia Maria de Sá Basilio Lins, JoseteLuziaLeite, Simone de Godoy, Patrícia dos Santos Claro Fuly, Silvia Teresa Carvalho de Araújo, Ítalo Rodolfo Silva. Validation of the adherence questionnaire for Brazilian chronic kidney disease patients under hemodialysis. Rev Bras Enferm [Internet]. 2017 MaiJun;70(3):558-65.

20. Sontakke S, Budania R, Bajait C, Jaiswal K, Pimpalkhute S. Evaluation of adherence to therapy in patients of chronic kidney disease. Indian J Pharmacol [serial online]. 2015 [cited2019Jan9]; 47:668-71.

21. Abdulmalik $M$ Alkatheri, Sarah $M$ Alyousif, Najla Alshabanah, Abdulkareem M Albekairy, Shemylan Alharbi, Fayze F Alhejaili, Abdullah A Alsayyari, Abeer MA Qandil, Amjad M Qandil. Medication adherence among adult patients on hemodialysis. Saudi J Kidney Dis Transpl. 2014;25(4):762-768.

22. Ronita J. Bland, Randall R Cottrell, Liliana R Guyler. Medication Compliance of Hemodialysis Patients and Factors Contributing to Non-Compliance. Dialysis \& Transplantation. May 2008;37(5):174-78. 